\title{
APPLIED OPTIMIZATION AND APPROXIMATION
}

\section{Octav Olteanu ${ }^{1}$}

${ }^{\prime}$ Department of Mathematics - Informatics, University Politehnica of Bucharest, Bucharest, Romania

\begin{abstract}
The present work deals with optimization in kinematics, generalizing previous results of the author. A second theme is maximizing the constrained gain linear function and minimizing the constrained cost function. Elementary notions of optimal control are considered as well. Finally, polynomial approximation results on unbounded subsets in several variables are applied to the moment problem. The existence of the solution of a two dimensional moment problem is characterized in terms of quadratic forms.
\end{abstract}

Keywords: Optimization, Kinematics, Gain function, Cost function, Approximation, Unbounded subsets, Markov moment problem.

Received: 10 July 2014/ Revised: 20 August 2014/ Accepted: 23 August 2014/ Published: 27 August 2014

\section{Contribution/ Originality}

The paper contributes the first logical analysis of the problems solved in the theorems 2.1, 2.2 (optimization in kinematics), 3.3 (minimization of the total cost), Lemma 4.2 (approximation), Theorem 4.3 (solving the moment problem). The paper's primary contribution is finding that suitable polynomial approximation yields solving the multidimensional moment problem. This study documents how practical and theoretical problems can be solved.

\section{INTRODUCTION}

The importance of increasing the kinetic energy of a system of particles is essential. On the other hand, we try to minimize the total constrained cost linear functional (of transportation, etc.) and to increase the constrained gain functional. Most of these problems can be solved by applying basic inequalities and studing the case when equalities occur in these inequalities. Such inequalities are: Cauchy-Schwarz-Buniakovski inequality, mean inequality, Hölder's inequality and many others. In practice, the extremum problems are usually constrained extremum problems. Some of the results in the present work have been presented during the Railway Stock National Symposium [1], Politehnica University of Bucharest. These results concern optimization in kinematics, respectively minimization and control of the total cost. Maximizing the gain is another subject briefly treated in this work. For basic notions of the present work, we 
mention the monographs [2-6], [7]. For applications of polynomial decomposition and approximation to the moment problem see [2], [8-13], [14-17]. An approximation method similar to ours, applied to the complex moment problem, appears in [9]. Most of these works are using Hahn-Banach type results in solving moment problems. For separation theorems applied to optimization, Mazur-Orlicz and moment problems see [5].

The paper is organized as follows. Sections 2 is devoted to optimization in kinematics. Section 3 contains elements of control and optimization of the total cost. The aim of Section 4 is to present an application of an $L^{1}$-approximation result to the multidimensional Markov moment problem. This leads to the characterization of the existence of the solution in terms of quadratic forms. Section 5 concludes the paper.

\section{OPTIMIZATION IN KINEMATICS}

Next we generalize some results from [1]. In the space $R^{n}$ we consider a system of $p$ material particles, of masses $m_{1}, \ldots, m_{p}$, which are moving following the action of a the continuous fields of forces

$$
\vec{f}_{1}, \ldots, \vec{f}_{p}, \vec{f}_{l}=\vec{f}_{l}(t), t \in\left[t_{0}, T\right], l=1, \ldots, p .
$$

The velocities $\vec{v}_{1}, \ldots, \vec{v}_{p}$ verify the following conditions

$$
\vec{v}_{l}\left(t_{0}\right)=\overrightarrow{0}, \vec{v}_{l} \in C^{(1)}\left([0, T], R^{n}\right), l=1, \ldots, p .
$$

2.1 Our first aim is determining conditions on the velocity-fields, such that the kinetic energy and the total kinetic moment of the system to be maximal. We assume that the euclidean norms in $R^{n}$ of the forces fields are bounded from above by the constants $M_{l}, l=1, \ldots, p$, so that by hypothesis we have:

$$
\left\|\vec{f}_{l}(t)\right\| \leq M_{l}, \forall t \in[0, T], l=1, \ldots, p
$$

Theorem-2.1.(i) If the movement of the system occurs such that the kinetic energy is maximal, the $\vec{f}_{l}$ is parallel to $\vec{v}_{l}$ at any moment of time, and we have

$$
\begin{aligned}
& v_{l, \max }(t)=\left(M_{l} / m_{l}\right) \cdot\left(t-t_{\mathrm{O}}\right), \quad l=1, \ldots, p, \\
& T_{\max }(t)=\frac{1}{2}\left(\sum_{l=1}^{p}\left(M_{l}^{2} / m_{l}\right)\right) \cdot\left(t-t_{\mathrm{O}}\right)^{2}, t \in\left[t_{\mathrm{O}}, T\right] .
\end{aligned}
$$

(ii) Under the additional assumption of a maximal kinetic momentum $\mathrm{M}=\mathrm{M}(t)$, we have: 


$$
\begin{aligned}
& v_{1}(t)=\cdots=v_{l}(t)=v(t), t \in[0, T], \quad M_{1} / m_{1}=\cdots=M_{p} / m_{p}, \\
& \mathrm{M}_{\max }(t)=\left(\sum_{l=1}^{p} m_{l}\right)\left(M_{k} / m_{k}\right)\left(t-t_{0}\right), k=1, \ldots, p .
\end{aligned}
$$

Proof: Following the Newton's second law, we have

$$
\begin{aligned}
& m_{l} \dot{\vec{v}}_{l}=\vec{f}_{l} \Rightarrow m_{l}\left\langle\dot{\vec{v}}_{l}, \vec{v}_{l}\right\rangle=\left\langle\vec{f}_{l}, \vec{v}_{l}\right\rangle \Rightarrow m_{l} \cdot d / d t\left((1 / 2)\left\|\vec{v}_{l}\right\|^{2}\right)=\left\langle\vec{f}_{l}, \vec{v}_{l}\right\rangle \Rightarrow \\
& \frac{1}{2} m_{l} v_{l}^{2}(t)=\int_{t_{0}}^{t}\left\langle\vec{f}_{l}(\tau), \vec{v}_{l}(\tau)\right\rangle d \tau \leq M_{l} \int_{t_{0}}^{t} v_{l}(\tau) d \tau, l=1, \ldots, p \Rightarrow \\
& T(t)=\frac{1}{2}\left(\sum_{l=1}^{p} m_{l} v_{l}^{2}(t)\right) \leq \sum_{l=1}^{p} M_{l} \int_{t_{0}}^{t} v_{l}(\tau) d \tau=T_{\max }(t) .
\end{aligned}
$$

Equality occurs in the last inequality if and only if

$$
\sum_{l=1}^{p}\left[M_{l} \int_{t_{0}}^{t} v_{l}(\tau) d \tau-(1 / 2) m_{l} v_{l}^{2}(t)\right]=0
$$

Using (3), we infer that each term of the sum (4) is nonnegative. Because the sum is vanishing, the conclusion is that in case of a maximal kinetic energy, each term is vanishing as well. Derivation with respect to $\boldsymbol{t}$ in the equalities obtained in this way leads to

$$
\begin{aligned}
& M_{l} v_{l}(t)=m_{l} v_{l}(t) \dot{v}_{l}(t), t \in[0, T] \Rightarrow v_{l, \text { optimal }}(t)=\left(M_{l} / m_{l}\right)\left(t-t_{0}\right) \\
& l=1, \ldots, p \Rightarrow T_{\max }(t)=\frac{1}{2}\left(\sum_{l=1}^{p}\left(M_{l}^{2} / m_{l}\right)\right) \cdot\left(t-t_{0}\right)^{2}
\end{aligned}
$$

So, the relations (1) follow, also applying Cauchy-Schwarz-Buniakovski inequality in $R^{n}$ and the case when equality occurs in this inequality. Next we additionally assume that the kinetic momentum

$$
\mathrm{M}(t)=\sum_{l=1}^{p} m_{l} v_{l}(t)=\sum_{l=1}^{p} \sqrt{m_{l}}\left(\sqrt{m_{l}} v_{l}(t)\right) \leq\left(\sum_{l=1}^{p} m_{l}\right)^{1 / 2}\left(2 \cdot T_{\max }(t)\right)^{1 / 2}
$$

is maximal too, at any moment of time $t$. Then we have equality in the above inequality. This happens if and only if there exists $\lambda(t)$ such that 


$$
\begin{aligned}
& m_{l} v_{l}^{2}(t)=\lambda(t) \cdot m_{l}, l=1, \ldots, p \Rightarrow v_{1, \max }(t)=\cdots=v_{p, \max }(t)=v(t)=\sqrt{\lambda(t)} \Rightarrow \\
& M_{1} / m_{1}=\cdots=M_{p} / m_{p} \Rightarrow \mathrm{M}_{\max }(t)=\left(\sum_{l=1}^{p} m_{l}\right) \cdot v_{\max }(t)=\left(\sum_{l=1}^{p} m_{l}\right) \cdot M_{k} / m_{k} \cdot\left(t-t_{0}\right),
\end{aligned}
$$

$k=1, \ldots, p$.

Now the relations (2) follow. This concludes the proof.

\subsection{Increasing the Momentum in a Concrete Case}

We next consider a method of increasing the "total momentum", illustrated in a particular case of a train formed by a locomotive and a wagon of masses $m_{1}, m_{2}$ respectively. The locomotive is coupled to the wagon with the aid of an elastic connection, of coefficient $k>0$. Let $F$ be the force applied to the locomotive and $\mu$ a friction coefficient. At the initial moment $t=t_{0}$, the velocities are assumed to be zero. We assume that the movement is rectilinear. From Newton's second low, the movement equations of the train follow:

$$
\begin{aligned}
& m_{1} \ddot{x}_{1}=F-k\left(x_{1}-x_{2}\right)-\mu g m_{1} \dot{x}_{1} \\
& m_{2} \ddot{x}_{2}=k\left(x_{1}-x_{2}\right)-\mu g m_{2} \dot{x}_{2},
\end{aligned}
$$

where $x_{1}, x_{2}$ are respectively the abscissa of the gravity centers of the two vehicles.

Theorem-2.2. The momentum $y=m_{1} \dot{x}_{1}+m_{2} \dot{x}_{2}$ is maximal if and only if

$$
\begin{aligned}
& \dot{x}_{1}(t)=\dot{x}_{2}(t), y_{\max }(t)=\left(m_{1}+m_{2}\right) \dot{x}_{1}(t)=\left(m_{1}+m_{2}\right) \dot{x}_{2}(t)= \\
& \left(m_{1}+m_{2}\right)^{1 / 2}(2 T(t))^{1 / 2}, T_{\text {optimal }}=\frac{y_{\max }^{2}(t)}{2\left(m_{1}+m_{2}\right)}= \\
& \frac{F_{\max }^{2}\left[1-\exp \left(-\mu g\left(t-t_{0}\right)\right)\right]^{2}}{2\left(m_{1}+m_{2}\right) \mu^{2} g^{2}} \rightarrow \frac{F_{\max }^{2}}{2\left(m_{1}+m_{2}\right) \mu^{2} g^{2}}, t \rightarrow \infty,
\end{aligned}
$$

where the force $F_{\max }$ is assumed constant with respect to $t$. It follows that the kinetic energy corresponding to the maximal momentum is increasing with $t$, having a horizontal asymptote at $\infty$.

Proof: Adding the two equations (5) one obtains a first order linear differential equation in the unknown function $y=m_{1} \dot{x}_{1}+m_{2} \dot{x}_{2}$ : 


$$
\dot{y}+\mu g y=F \Rightarrow y(t)=\exp (-\mu g t) \int_{t_{0}}^{t} F(x) \exp (\mu g x) d x .
$$

On the other hand, application to the sum of the moments of Schwarz inequality yields:

$$
y=\sum_{j=1}^{2} m_{j} \dot{x}_{j}=\sum_{j=1}^{2} \sqrt{m_{j}}\left(\sqrt{m_{j}} \dot{x}_{j}\right) \leq\left(\sum_{j=1}^{2} m_{j}\right)^{1 / 2}\left(\sum_{j=1}^{2} m_{j} \dot{x}_{j}^{2}\right)^{1 / 2},
$$

and equality occurs if and only if

$$
\left(\sqrt{m_{j}} \cdot \dot{x}_{j}\right)_{j=1}^{2}=\lambda\left(\sqrt{m_{j}}\right)_{j=1}^{2}
$$

for some $\lambda=\lambda(t)$. It follows that the relations from below hold

$$
\begin{aligned}
& \dot{x}_{1}(t)=\dot{x}_{2}(t)=\lambda(t), t \geq t_{0} \Rightarrow y_{\max }(t)=\left(m_{1}+m_{2}\right) \dot{x}_{j}(t)= \\
& \left(m_{1}+m_{2}\right)^{1 / 2}(2 T(t))^{1 / 2}, j=1,2, T(t):=(1 / 2)\left(\sum_{j=1}^{2} m_{j} \dot{x}_{j}^{2}(t)\right)
\end{aligned}
$$

For $F(x)=F_{\max }=$ const, from (6) and (7) we infer that

$$
T_{\text {optimal }}(t)=\frac{y_{\max }^{2}(t)}{2\left(m_{1}+m_{2}\right)}=\frac{F_{\max }^{2}\left[1-\exp \left(-\mu g\left(t-t_{0}\right)\right)\right]^{2}}{2\left(m_{1}+m_{2}\right) \mu^{2} g^{2}}
$$

Now the conclusion follows.

\section{MAXIMIZING THE GAIN AND THE CONTROL OF THE COST}

Proposition 3.1. Under the constraints

$\sum_{j=1}^{n} x_{j}^{p} \leq M, c_{j}>0 \forall j=1, \ldots, n$ for some $p>1$ the gain $G$ is maximal if and only if there exists

$\lambda>0$ such that

$$
\begin{aligned}
& x_{j}^{p}=\lambda c_{j}^{q}, j=1, \ldots, n, 1 / p+1 / q=1, \\
& \lambda=M /\left(\sum_{j=1}^{n} c_{j}^{q}\right), G_{\max }=M^{1 / p}\left(\sum_{j=1}^{n} c_{j}^{q}\right)^{1 / q} .
\end{aligned}
$$

Proof: Hőlder’s inequality yields

$$
G=\sum_{j=1}^{n} c_{j} x_{j} \leq\left(\sum_{j=1}^{n} c_{j}^{q}\right)^{1 / q}\left(\sum_{j=1}^{n} x_{j}^{p}\right)^{1 / p} \leq M^{1 / p}\left(\sum_{j=1}^{n} c_{j}^{q}\right)^{1 / q}
$$

and equality occurs in the first relation if and only if there exists 


$$
\lambda>0, x_{j}^{p}=\lambda c_{j}^{q}, j=1, \ldots, n,
$$

(following the proof of the Hőlder's inequalitiy and the associated remark [7]). Now the conclusion follows and the proof is complete.

Next we study the constrained extreme values of the total cost, also using elementary notions of optimal control. We start by determining the maximum of the total cost, under a constraint on the quantities of the goods. Such problems are useful in determining an upper bound that stands for a threshold. If the coefficients $c_{j}$ stand for gains, one obtains a maximization of the total gain. Let consider the constrained linear form

$$
f\left(x_{1}, \ldots, x_{n}\right)=\sum_{j=1}^{n} c_{j} x_{j}, \sum_{j=1}^{n} \alpha_{j}^{2} x_{j}^{2}=M^{2},
$$

where $c_{j} \geq 0$ are given constants standing for the cost or gain of each unit of good, $x_{j}$ being the quantities (the number of the good - units) that are going to be transported (respectively sold) following the unitary costs $c_{j}, j=1, \ldots, n$. Assume that

$$
\alpha_{j} \neq 0, j=1, \ldots, n
$$

We are going to determine the optimal quantities, such that the total cost (respectively gain) to be maximal, under the elliptical constrained on $x_{j}$, written above. Following the elementary theory of conditional extremum, we define the Lagrange's function $L\left(x_{1}, \ldots, x_{n}, \lambda\right)=\sum_{j=1}^{n} c_{j} x_{j}+\lambda\left(\sum_{j=1}^{n} \alpha_{j}^{2} x_{j}^{2}-M^{2}\right) ; \frac{\partial L}{\partial x_{j}}=0 \Leftrightarrow$

$c_{j}=-2 \lambda \alpha_{j}^{2} x_{j, o p t i m}, j=1, \ldots, n \Rightarrow x_{j, \text { optim }}=-c_{j} /\left(2 \lambda \alpha_{j}^{2}\right)=x_{j}+u_{j} \Rightarrow$

$\lambda<0, M^{2}=\sum_{j=1}^{n} \alpha_{j}^{2} x_{j, \text { optim }}^{2}=\frac{1}{4 \lambda^{2}}\left(\sum_{j=1}^{n} c_{j}^{2} / \alpha_{j}^{2}\right) \Rightarrow-\frac{1}{2 \lambda}=\frac{M}{\left(\sum_{j=1}^{n} c_{j}^{2} / \alpha_{j}^{2}\right)^{1 / 2}}$

$u_{j}=x_{j, \text { optim }}-x_{j}=-\frac{c_{j}}{2 \lambda \alpha_{j}^{2}}-x_{j}=\frac{M c_{j}}{\left(\sum_{j=1}^{n} c_{j}^{2} / \alpha_{j}^{2}\right)^{1 / 2} \alpha_{j}^{2}}-x_{j} ;$

$M^{2}=\sum_{j=1}^{n} \alpha_{j}^{2} x_{j}^{2}=\sum_{j=1}^{n} \alpha_{j}^{2} x_{j, o p t i m}^{2}=\sum_{j=1}^{n} \alpha_{j}^{2}\left(x_{j}+u_{j}\right)^{2} \Rightarrow$

$\mathrm{o}=\sum_{j=1}^{n} \alpha_{j}^{2} u_{j}\left(2 x_{j}+u_{j}\right) \Rightarrow \vec{u} \perp\left(\alpha_{j}^{2}\left(x_{j, \text { optim }}+x_{j}\right)\right)_{j=1}^{n} ;$

$f_{\max }=f\left(\left(x_{j, \text { optim }}\right)_{j=1}^{n}\right)=-\frac{1}{2 \lambda}\left(\sum_{j=1}^{n} c_{j}^{2} / \alpha_{j}^{2}\right)=$

$\frac{M}{\left(\sum_{j=1}^{n} c_{j}^{2} / \alpha_{j}^{2}\right)^{1 / 2}}\left(\sum_{j=1}^{n} c_{j}^{2} / \alpha_{j}^{2}\right)=M\left(\sum_{j=1}^{n} c_{j}^{2} / \alpha_{j}^{2}\right)^{1 / 2}$. 
Theorem-3.2. The above equalities give the optimal quantities $x_{j, o p t i m}$, the controls $u_{j}$ and the maximum value $f_{\max }$ of the cost (respectively gain) function.

Next we go on with a constrained minimization problem of the total cost, which follows from the mean inequality [7]. We recall this problem due to its simplicity and importance in applications. Let consider the constrained minimization problem

$$
\min \left(\sum_{j=1}^{n} c_{j} x_{j}\right), c_{j}>0, x_{j} \geq 0, j=1, \ldots, n, \prod_{j=1}^{n} x_{j}:=P_{2}=\text { const } .
$$

Application of the mean inequality [7] lieds to the following result.

Theorem 3.3. The minimum of the total cost, constrained by condition $\prod_{j=1}^{n} x_{j}=P_{2}=$ const. is realized for equal values of the products $c_{j} x_{j}, j=1, \ldots, n$. Namely, we have

$$
x_{j, o p t .}=\sqrt[n]{P_{1} P_{2}} / c_{j}, j=1, \ldots, n, \quad \min \left\{\sum_{j=1}^{n} c_{j} x_{j} ; \prod_{j=1}^{n} x_{j}=P_{2}\right\}=n \cdot\left(\sqrt[n]{P_{1} P_{2}}\right) .
$$

Proof: Because both products $P_{1}, P_{2}$ are constants, denoting $y_{j}=c_{j} x_{j}, j=1, \ldots, n$, from the mean inequality and the case when equality occurs, we infer that the minimum of the sum of the $y_{j}{ }^{\prime} s, j=1, \ldots, n$ is realized for equal values of of $y_{j}, j=1, \ldots, n$ :

$$
\begin{gathered}
y_{j}>0, \prod_{j=1}^{n} y_{j}=P_{1} P_{2}=\text { const. } \Rightarrow \min \left\{\sum_{j=1}^{n} y_{j} ; \prod_{j=1}^{n} y_{j}=P_{1} P_{2}\right\}=n \cdot \sqrt[n]{P_{1} P_{2}}, \\
y_{1}=y_{2}=\cdots=y_{n}=\sqrt[n]{P_{1} P_{2}} .
\end{gathered}
$$

Following our notations, the conclusion follows.

\section{POLYNOMIAL APPROXIMATION ON UNBOUNDED SUBSETS AND THE MULTIDIMENSIONAL MOMENT PROBLEM}

Recall that in several real dimensions, there exist positive polynomials on $\mathbb{R}_{+}^{n}$ that are not writable in terms of sums of squares. Consequently, "computable" characterizations for the existence of the solution might be difficult. We solve this difficulty by using an approximation process of nonnegative compactly supported continuous functions by sums of tensor products of positive polynomials on the whole $R_{+}$, in each separate variable. 
Theorem-4.1. (see [16]) Let $A \subset R^{n}$ be a closed unbounded subset and $v$ a positive regular $M$ - determinate Borel measure [7] on A, with finite moments of all orders. Then for any $\psi \in\left(C_{0}(A)\right)_{+}$, there is a sequence $\left(p_{m}\right)_{m}$ of polynomials on $A, p_{m} \geq \psi, p_{m} \rightarrow \psi$ in $L_{v}^{1}(A)$. We have

$$
\lim \int_{A} p_{m} d v=\int_{A} \psi d v
$$

the cone $P_{+}$of positive polynomials is dense in $\left(L_{v}^{1}(A)\right)_{+}$and $P$ is dense in $L_{v}^{1}(A)$.

Recall that a determinate (M-determinate) measure is uniquely determinate by its moments (or by its values on polynomials).

Lemma-4.2. The convex cone of sums of tensor products $p_{1} \otimes p_{2}$ of positive polynomials in separate variables $t_{1}, t_{2}$ is dense in $\left(C_{c}\left(R_{+}^{2}\right)\right)_{+}$, the cone of all nonnegative continuous compactly supported functions, with their support contained in $R_{+}^{2}$, with respect to the $L_{v}^{1}-$ norm, for any measure $v=v_{1} \times v_{2}$, where $v_{j}, j=1,2$ are positive regular $M-$ determinate Borel measures on $R_{+}$, with finite moments of all natural orders.

Proof: If $K$ is the support of a function $f \in C_{c}\left(R_{+}^{2}\right), f \geq 0$, then

$$
K \subset K_{1} \times K_{2}, K_{j}=p r_{j}(K), j=1,2
$$

Consider a rectangle $R_{2}$ containing the above Cartesian product of compacts and apply approximation of the extension of $f$ vanishing on the rectangle outside its support, by Luzin's theorem. Next, approximate this continuous function on the rectangle by the corresponding Bernstein polynomials in two variables. Each term of such a polynomial is a tensor product $p_{1} \otimes p_{2}$, of positive polynomials in each variable. Extend each $p_{j}$ such that it vanishes outside $p r_{j}\left(R_{2}\right)$, applying then Luzin's theorem once more, this time in one variable, $j=1,2$. This procedure does not change the values of $p_{j}$ on $K_{j}, j=1,2$. One obtains approximation by sums of tensor products of positive continuous functions with compact support, in each variable $t_{j}, j=1,2$. The approximating process holds in $L^{1}$ norm and uniformly on $K$. Next 
one approximates each of these function via Theorem 4.1, in each separate variable, in the space $L_{v_{j}}^{1}\left(R_{+}\right), j=1,2$, by means of positive polynomials on the nonnegative semi axes: one applies Theorem 4.1 for $\mathrm{n}=1, \mathrm{~A}=\mathrm{R}_{+}$. This concludes the proof.

Let $Y$ be an order complete Banach lattice with solid norm.

Theorem-4.3. Let $v=v_{1} \times v_{2}$ be a product measure on $H=R_{+}^{2}$, where $v_{k}, k=1,2$ are regular positive $M$ - determinate Borel measures [7] with finite moments of all natural orders on $R_{+}$. Let $(y(j, k))_{(j, k) \in \mathrm{N}^{2}}$ be a sequence in $Y, F_{2}: X=L_{v}^{1}\left(R_{+}^{2}\right) \rightarrow Y$ a linear positive bounded operator. The following statements are equivalent:

(a) there is a unique linear positive bounded operator $F \in B(X, Y)$ which verifies the moment conditions

$$
F\left(\varphi_{j, k}\right)=y(j, k), \varphi_{j, k}\left(t_{1}, t_{2}\right):=t_{1}^{j} t_{2}^{k}, \forall(j, k) \in \mathrm{N}^{2}
$$

and $F$ is dominated by $F_{2}$ on the positive cone of $X,\|F\| \leq\left\|F_{2}\right\|$;

(b) for any finite subsets $J_{1}, J_{2} \subset \mathrm{N}$ and all

$$
\left\{a_{j}\right\}_{j \in J_{1}} \subset R,\left\{b_{m}\right\}_{m \in J_{2}} \subset R
$$

one has:

$$
\begin{aligned}
& 0 \leq \sum_{\substack{i, j \in J_{1}, m, n \in J_{2}}} a_{i} a_{j} b_{m} b_{n} y(i+j+k, m+n+p) \leq \sum_{\substack{i, j \in J_{1} \\
m, n \in J_{2}}} a_{i} a_{j} b_{m} b_{n} F_{2}\left(\varphi_{i+j+k, m+n+p}\right), \\
& (k, p) \in\{0,1\} \times\{0,1\} .
\end{aligned}
$$

Proof: The implication $(a) \Rightarrow(b)$ is almost obvious. In order to prove the converse, one uses the density of sums of tensor products of positive polynomials in each one separate variable in the positive cone of $C_{c}\left(R_{+} \times R_{+}\right)$(Lemma 4.2), in approximating nonnegative continuous compactly supported functions. Using the analytic expression of positive polynomials on the positive semiaxes [2], one observes that $(b)$ says that the linear operator $F_{0}$ defined on polynomials and verifying the moment conditions is positive and dominated by $F_{2}$ on the cone generated by tensor products of positive polynomials in separate variables $t_{1} \in R_{+}$, respectively 
$t_{2} \in R_{+}$. In particular, this linear operator has a positive extension $F$, to the space of all integrable functions dominated in absolute value by a polynomial, on $R_{+}^{2}$ (see Cristescu $1976 \mathrm{p}$. 160, [4]). This space contains the space of continuous compactly supported functions. Let $\psi$ be a nonnegative continuous compactly sopported function, with the support contained in $\mathrm{R}_{+}^{2}$. By the preceding remarks, one approximates $\psi$ on a rectangle containing $p r_{1}(\sup p o r t \psi) \times p r_{2}(\sup p o r t \psi)$ by means of Luzin's theorem and the corresponding Bernstein polynomials in two variables. Then one approximates $\psi$ by sums of tensor products of positive polynomials in each separate variables:

$$
\sum_{j=0}^{k(m)} p_{m, 1 . j} \otimes p_{m, 2, j} \rightarrow \psi, m \rightarrow \infty
$$

in the space $L_{V}^{1}\left(R_{+} \times R_{+}\right)$. Using (b) and applying Fatou's lemma, one obtains:

$$
\begin{aligned}
& 0 \leq y^{*}(F(\psi)) \leq \liminf _{m}\left(y^{*} \circ F\right)\left(\sum_{j=0}^{k(m)} p_{m, 1, j} \otimes p_{m, 2, j}\right) \leq \\
& \lim _{m}\left(y^{*} \circ F_{2}\right)\left(\sum_{j=0}^{k(m)} p_{m, 1, j} \otimes p_{m, 2, j}\right)= \\
& y^{*}\left(F_{2}(\psi)\right), \psi \in\left(C_{c}\left(R_{+} \times R\right)\right)_{+}, \forall y^{*} \in Y_{+}^{*} .
\end{aligned}
$$

Assume that

$$
F_{2}(\psi)-F(\psi) \notin Y_{+}
$$

Using a separation theorem, it should exist a positive linear continuous functional $y^{*} \in Y_{+}^{*}$ such that

$$
y^{*}\left(F_{2}(\psi)-F(\psi)\right)<0
$$

that is $y^{*}\left(F_{2}(\psi)\right)<y^{*}(F(\psi))$. This relation contradicts (8). The conclusion is that we must have

$$
F(\psi) \leq F_{2}(\psi), \psi \in\left(C_{c}\left(R_{+} \times R_{+}\right)\right)_{+} .
$$

Then for arbitrary $g \in C_{c}\left(R_{+} \times R_{+}\right)$one writes

$$
|F(g)| \leq F_{2}\left(g^{+}\right)+F_{2}\left(g^{-}\right)=F_{2}(|g|) \Rightarrow\|F(g)\| \leq\left\|F_{2}\right\| \cdot\|g\|_{1} .
$$


The conclusion is that the operator $F$ is positive and continuous, of norm dominated by

$\left\|F_{2}\right\|$, on a dense subspace of $L_{v}^{1}\left(R_{+} \times R_{+}\right)$. It has a unique linear extension preserving its qualities. This concludes the proof.

\section{CONCLUSIONS}

We have solved some optimization problems with practical meaning, by using well - known inequalities and the extreme cases when equality occurs. These extreme values coincide with the extremes of our functions, having a practical meaning. For example, Theorem 3.3 says that (under some constraints), the optimal quantities are converse - proportional with respect to the corresponding (transport) costs. This result has a very natural practical meaning. The aim of section 4 is to solve the difficulty created by the fact that in several dimensions there are positive polynomials that are not writable by means of sums of squares. Using an approximation method, we obtain the characterization for the existence of the solution of the multidimensional moment problem in terms of "computable" quadratic forms.

The author declares that there is no conflict of interests regarding the publication of this paper.

Funding: This study received no specific financial support.

Competing Interests: The author declares that there are no conflicts of interests regarding the publication of this paper.

\section{REFERENCES}

[1] O. Olteanu, "Kinematics and optimization in railway transport", National Railway Stock Symposium, X - th Edition, Presented Works, November, 23-24 2012, Matrix Rom, Bucharest, (In Romanian), 2012.

[2] N. I. Akhiezer, The classical moment problem and some related questions in analysis. Edinburgh and London: Oliver and Boyd, 1965.

[3] V. I. Arnold, Mathematical methods of classical mechanics, 2nd ed. New York: Springer, 1989.

[4] R. Cristescu, Ordered vector spaces and linear operators. Ed. Tunbridge Wells, Kent: Academiei, Bucharest and Abacus Press, 1976.

[5] R. B. Holmes, Geometric functional analysis and its applications. New York: Springer-Verlag Inc., 1975 .

[6] S. Boyd, and L. Vandenberghe, Convex optimization: Cambridge University Press, 2004, 2009.

[7] W. Rudin, Real and complex analysis, 3rd ed.: McGraw - Hill, Inc., 1987.

[8] J. Cassier, "Problème des moments sur un compact de Rn et décomposition des polynômes à plusieurs variables," J. Funct. Anal., vol. 58, pp. 254-266, 1984.

[9] L. L. Lemnete-Ninulescu and A. Zlătescu, "Some new aspects of the L moment problem," Rev. Roumaine Math. Pures Appl., vol. 55, pp. 197-204, 2010. 
[10] J. M. Mihăilă, O. Olteanu, and C. Udrişte, "Markov-type moment problems for arbitrary compact and for some non-compact Borel subsets of Rn," Rev. Roumaine Math. Pures Appl., vol. 52, pp. 655664, 2007.

[11] A. Olteanu and O. Olteanu, "Some unexpected problems of the moment problem," in Proc. of the Sixth Congress of Romanian Mathematicians, Ed. Academiei, Vol. I (Scientific Contributions), Bucharest, 2009, pp. 347-355.

[12] O. Olteanu, "Application de théorèmes de prolongement d'opérateurs linéaires au problème des moments et à une généralisation d'un théorème de Mazur-Orlicz," C. R. Acad. Sci. Paris 313, Série I, pp. 739-742, 1991.

[13] O. Olteanu, Geometric aspects in operator theory and applications. Saarbrücken: Lambert Academic Publishing, 2012.

[14] O. Olteanu, "Moment problems on bounded and unbounded domains," International Journal of Analysis, Article ID 524264. Available: http://dx.doi.org/10.1155/2013/524264, vol. 2013, p. 7, 2013.

[15] O. Olteanu, "New results on Markov moment problem," International Journal of Analysis, Article ID 901318. Available: http://dx.doi.org/10.1155/2013/901318, vol. 2013, p. 17, 2013.

[16] O. Olteanu, "Approximation and Markov moment problem on concrete spaces," Rendiconti del Circolo Matematico di Palermo, vol. 63, pp. 161-172, 2014.

[17] M. Putinar and F. H. Vasilescu, "Problème des moments sur les compacts semi-algébriques," C. R. Acad. Sci. Paris, 323, Série I, pp. 787-791, 1996. 